Article

\title{
Super Dielectric Materials
}

\section{Samuel Fromille and Jonathan Phillips *}

Physics Department, Naval Postgraduate School, Monterey, CA 93943, USA;

E-Mail: sfromille@gmail.com

* Author to whom correspondence should be addressed; E-Mail: jphillip@ nps.edu.

External Editor: Christof Schneider

Received: 6 November 2014; in revised form: 26 November 2014 / Accepted: 12 December 2014 / Published: 22 December 2014

\begin{abstract}
Evidence is provided here that a class of materials with dielectric constants greater than $10^{5}$ at low frequency $\left(<10^{-2} \mathrm{~Hz}\right)$, herein called super dielectric materials (SDM), can be generated readily from common, inexpensive materials. Specifically it is demonstrated that high surface area alumina powders, loaded to the incipient wetness point with a solution of boric acid dissolved in water, have dielectric constants, near $0 \mathrm{~Hz}$, greater than $4 \times 10^{8}$ in all cases, a remarkable increase over the best dielectric constants previously measured for energy storage capabilities, ca. $1 \times 10^{4}$. It is postulated that any porous, electrically insulating material (e.g., high surface area powders of silica, titania, etc.), filled with a liquid containing a high concentration of ionic species will potentially be an SDM. Capacitors created with the first generated SDM dielectrics (alumina with boric acid solution), herein called New Paradigm Super (NPS) capacitors display typical electrostatic capacitive behavior, such as increasing capacitance with decreasing thickness, and can be cycled, but are limited to a maximum effective operating voltage of about $0.8 \mathrm{~V}$. A simple theory is presented: Water containing relatively high concentrations of dissolved ions saturates all, or virtually all, the pores (average diameter $500 \AA$ ) of the alumina. In an applied field the positive ionic species migrate to the cathode end, and the negative ions to the anode end of each drop. This creates giant dipoles with high charge, hence leading to high dielectric constant behavior. At about $0.8 \mathrm{~V}$, water begins to break down, creating enough ionic species to "short" the individual water droplets. Potentially NPS capacitor stacks can surpass "supercapacitors" in volumetric energy density.
\end{abstract}

Keywords: capacitor; dielectric; energy 


\section{Introduction}

There are several distinct capacitor technologies, and for understanding the import of the present work it is helpful to compare/contrast two types; "supercapacitors", and traditional electrostatic (or "ceramic") capacitors [1]. In essence, supercapacitors are electrochemical cells with an extremely high electrode surface area, and hence are more properly known as electrode double layer capacitors (EDLC). EDLC increase capacitance by increasing the electrically conductive electrode surface area, that is the size of the double layer where the charge is stored.

Most of the volume of an EDLC is the high surface area electrode in the form of electrically conductive powder, a material for which "dielectric constant" is a meaningless parameter. Increasing the EDLC volume while maintaining the shape/surface area of the attached ends constant, increases the amount of electrode material area, concomitantly increasing the capacitance in direct proportion to the volume increase. Hence, EDLC/supercapacitor performance is generally reported as "per gram" or per unit volume. This explains the recent interest in employing graphene in EDLC/supercapacitors [2-4], as graphene is arguably the "ultimate" material for creation of high surface area electrodes. Indeed, it has a very high electrical conductivity and the measured surface of some graphene forms are near the theoretical limit $\left(\sim 2700 \mathrm{~m}^{2} / \mathrm{g}\right)$. Once the best EDLC/supercapacitors incorporate graphene, supercapacitor energy density will be near a theoretical limit, hence further significant energy density increase is unlikely.

In contrast to EDLC, the capacitance of a traditional electrostatic capacitor with constant sized electrodes decreases with volume. The material between the electrodes is an electrical insulator, and this material is characterized by a dielectric constant. In an electrostatic capacitor, the capacitance is inversely proportional to the distance between the plates. Given plates of a constant size, the thinner an electrostatic capacitor is, the greater the capacitance. Clearly, the means to improve the performance of this style of capacitor is either to make them thinner, find materials with higher dielectric constants. Or materials with a very high breakdown voltage.

Relative to EDLC improvements, the ultimate energy density of traditional electrostatic capacitors has been modest over the last few decades. The biggest improvement has resulted from new techniques that permit the fabrication of thinner ceramic dielectric layers. Enhancements in the dielectric constant of the best ceramic have been more modest. Much of the effort to increase the dielectric constant has focused for decades on improving one material, barium titanate [5-9]. Additionally, there have been efforts to create "colossal dielectric" materials [10-14], some of which reportedly have "extrinsic" dielectric constants of the order of $10^{5}$.

Herein, we introduce a novel hypothesis for a class of super dielectric materials (SDM), that is materials with (intrinsic) dielectric constants greater than $10^{5}$ at very low frequency, and provide test data demonstrating the existence of one SDM material. The hypothesis: Charged species in liquid drops in the pores of solids will migrate to create dipoles, equal in size to the drops, in an applied electric field. This phenomenology can be manipulated to create a high dielectric material, potentially to be deployed in a new generation of electrostatic capacitors, so called New Paradigm Super (NPS) capacitors. These materials will be designed and tested on the basis of the following "application postulate": Adding solutions containing ions (e.g., acid solutions) to highly porous insulating materials creates a high dielectric, or even "superdielectric" (dielectric constant $>10^{5}$ ), material. 
Simple studies reported herein suggest this hypothesis is correct, and that the proposed application postulate works in practice. Specifically, the measured dielectric constant of one example, high surface area alumina incorporating a solution of boric acid, is orders of magnitude higher (ca. $\sim 10^{9}$ ) than any form of barium titanate (ca. $\sim 10^{3}$ ). It is in fact an SDM. The high dielectric constants measured suggest a path forward to developing a classic electrostatic capacitor as an alternative to supercapacitors for electrical energy storage/power delivery.

The results also reveal a limitation to the initial design: The electrolyte "breaks down", resulting in conduction, just as in a superconductor, at a relatively low voltage [15]. For the particular materials combination reported here the ultimate "capacitive" voltage was about $0.8 \mathrm{~V}$. Still, extrapolating the current results to a "depth" typical of ceramic capacitors ( 5 micron), and assuming better electrolytes will yield a higher ultimate voltage $(2.5 \mathrm{~V})$, yield an energy density for NPS capacitors of the order $1000 \mathrm{~J} / \mathrm{cm}^{3}$, a value several times higher than that of the best commercial supercapacitor. In fact, these computations are conservative; for example commercial ceramic capacitors with dielectric thickness of $<1 \mu$ are now available, even in large stacks [16].

\section{Experimental Section}

Dielectric Fabrication: The materials employed to create the specific dielectric employed in this study; alumina/boric acid solution super dielectric material (A-SDM); were high surface area aluminum oxide powder (Alfa Aesar, Ward Hill, MA, USA; $\gamma$-phase; 99.97\%; 3 micron APS Powder; S.A. $80-120 \mathrm{~m}^{2} / \mathrm{g}$; CAS 1344-28-1); boric acid powder (BDH; 99.5\% $\mathrm{H}_{3} \mathrm{BO}_{3}$; CAS 10043-35-3); and distilled deionized water. These constituents were mixed by hand in this ratio in all cases: $1 \mathrm{~g}$ alumina: $1 \mathrm{~mL} \mathrm{H} \mathrm{H}_{2} \mathrm{O}$ : $0.1 \mathrm{~g}$ boric acid powder. This created a spreadable paste with no "free" water (incipient wetness). It is interesting to note that a mixture of $1 \mathrm{~mL}$ of water and $0.1 \mathrm{~g}$ boric acid is only weakly acidic with a theoretical $\mathrm{pH}$ of approximately 4.5 .

As pore structure is a significant component of the proposed model, the surface area and pore structure were determined from BET nitrogen isotherms collected at $77{ }^{\circ} \mathrm{K}$ and analyzed using a Quantachrome NOVA 4200e (Boynton Beach, FL, USA). Two samples were independently measured and both yielded results within $5 \%$ for all parameters; specifically a surface area of $39 \pm-1 \mathrm{~m}^{2} / \mathrm{g}$, a total pore volume of $0.45 \mathrm{~cm}^{3} / \mathrm{g}$ and an average pore radius of $245 \pm-3 \AA$.

The dielectric paste was spread evenly on a $5 \mathrm{~cm}$ diameter disc of GTA grade Grafoil $(0.76 \mathrm{~mm}$ thick, $>99.99 \%$ carbon). As described elsewhere [17,18] Grafoil is a commercially available high purity carbon material (available in sheets or rolls) made by compressing naturally occurring graphite flakes with a surface area of the order of $20 \mathrm{~m}^{2} / \mathrm{g}$. In the final step a second sheet of Grafoil is placed on top, and the thin "capacitor" then mechanically pressed to create a near constant thickness as determined by measurements made at multiple positions using a hand held micrometer. The "effective thickness" of the dielectric used in all computations herein was based on subtracting the thickness of the Grafoil sheets from the measured gross thickness of the capacitor.

Once constructed the capacitors were placed in an electrically insulating plastic jig with bottom and top cylindrical aluminum electrodes of $5 \mathrm{~cm}$ diameter and $5 \mathrm{~mm}$ thickness. A $250 \mathrm{~g}$ weight was placed on top in all cases. These capacitors were then placed in simple circuits (Figure 1) for measurements of charge and discharge and extrapolation of the data to energy storage information. It is important to 
note that charging and discharging were generally done through several different resistors, specifically nominal 528, 99, or $20.1 \mathrm{kOhm}$ resistors. The smallest was used in order to speed up processes, such as for multi-cycle tests, and for the thin capacitors in which the capacitance was greater than 50 milliFarads.

Figure 1. Measuring capacitive properties. (a) With the switch "down" the discharge voltage across the capacitor is measured, and with the switch "up" the voltage across the capacitor during charging is recorded; (b) the capacitor is placed in a hard plastic jig between two aluminum electrodes with diameter of $5 \mathrm{~cm}$; (c) a picture of the completed capacitor with the aluminum/water/boric acid paste squeezed between two sheets of Grafoil.

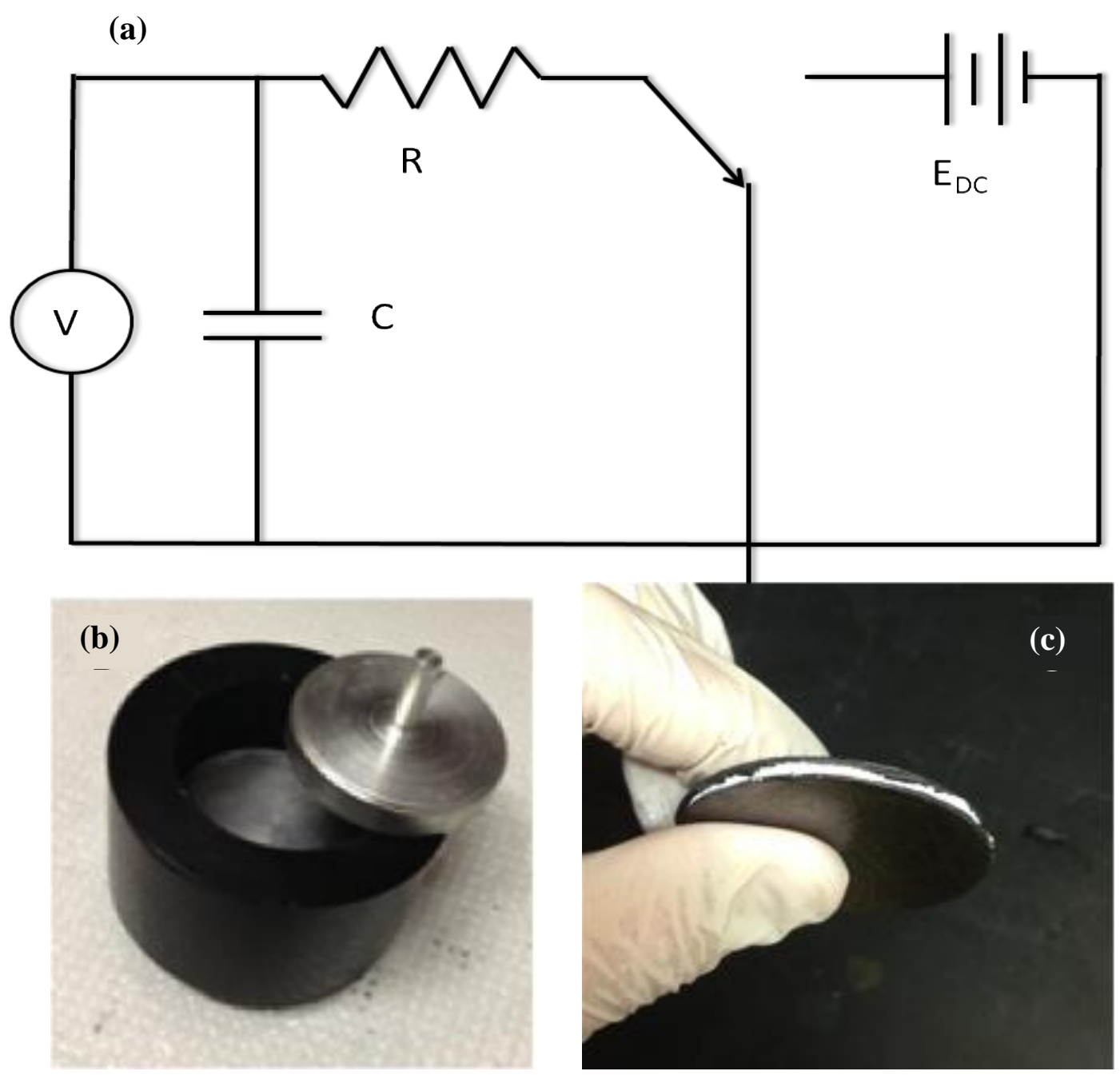

Measurement: The method employed to measure the capacitance for this study was the classic "RC time constant" approach. This method was selected because it is the only one suited for the measurement of energy storage, the most likely application of the SDM. Indeed, there is considerable controversy regarding the energy storage capabilities of electrostatic (ceramic) capacitors. Recently it was cogently argued that capacitance data improperly extrapolated without regard to saturation, maximum voltage, operating voltage and other factors [19], can lead to exaggerated estimates of the energy density for ferroelectric based capacitors. The RC time constant approach was taken herein as it 
provides a direct measure of the total energy output from the capacitor. There is no need to extrapolate data, or make assumptions.

There are several ways to measure capacitance, each with advantages and disadvantages. Alternatives to the RC time constant approach for measuring capacitance, and concomitantly dielectric constant, include multi-meters with a "capacitance" setting, and Impedance Spectroscopy (IS). It is critical to understand that these methods are single voltage methods.

For both multi-meters and IS, at the selected "bias" voltage, generally zero, small swings in voltage are analyzed (essentially RC time constant method). In order to avoid errors due to thermal effects, the tolerable voltage swings in IS are very small, of the order of $25 \mathrm{mV}$ at ambient [20,21]. In particular, IS is clearly the best method for impedance determination above $\sim 20 \mathrm{~Hz}$, but it does not provide direct measure of energy storage. It does not even provide information on the maximum operating voltage.

The primary test platform was a National Instruments ELVIS II electronics prototyping board implemented with LabView 2011 software. An additional multimeter, Agilent U1252A, was used for independent parameter verification. It is further notable that the capacitance of several types of commercial capacitors were measured using the above described instruments and protocol, and in every case the measured value and the listed value were within $30 \%$.

\section{Results}

Basic phenomenological data from studies of cyclic charging and discharging show that capacitors employing A-SDM behave nearly as ideal capacitors over a limited, repeatable, voltage range. Typical multi-cycle data from one NPS capacitor, charged through a $99 \mathrm{kOhm}$ resistor from a power supply operated at $4 \mathrm{~V}$, then discharged through the same resistor, is shown in Figure 2. Re-plotting similar data from several NPS capacitors using A-SDM dielectric of different thicknesses, and discharged through a $528 \mathrm{kOhm}$ resistor, was done to test the proposition that these capacitors charge/discharge exponentially, as per standard electrostatic capacitors:

$$
\ln \left(\mathrm{V} / \mathrm{V}_{0}\right)=\mathrm{t} / \mathrm{RC}
$$

Moreover, the dielectric constant can be obtained from the time constant and this standard equation:

$$
C=\varepsilon_{0} \varepsilon_{R} \frac{A}{d}
$$

where $\varepsilon_{0}$ is the permittivity of free space $\left(8.85 \times 10^{-12} \mathrm{~F} / \mathrm{m}\right)$ and $\varepsilon_{R}$ is the dielectric constant. The area of the plate surface is $A$ and the distance between the two electrode surfaces is $d$.

From the plots of one multi-cycle data set (Figure 2) it is clear that in all cases below about $0.8 \mathrm{~V}$ at very low frequency the A-SDM have nearly constant time constants, hence constant capacitance. Using these measured time constants, the resistance value, and the physical parameters of the capacitors the dielectric constants were computed for all three charge and discharge cycles. Specifically, for the first discharge cycle the dielectric constant was $\sim 1.1 \times 10^{9}$, but it roughly doubled by the third cycle. The first charge cycle showed a dielectric constant of $\sim 1.0 \times 10^{9}$, but the last charge cycle dielectric constant was roughly only one third of that value. Employing even the lower first cycle value indicates that the A-SDM material is a super dielectric material. Its dielectric constant is orders of magnitude greater than that required by the definition of SDM: materials possessing a dielectric constant $>10^{5}$. 
Figure 2. Capacitive cycling. These capacitors go through regular cycles, as shown. Note that the discharge time during the steady "capacitive part" of the cycles (below $\sim 0.8 \mathrm{~V}$ ) for a $99 \mathrm{kOhm}$ resistor) is more than $2000 \mathrm{~s}$. There is some difference between charge and discharge cycles in terms of apparent capacitance.

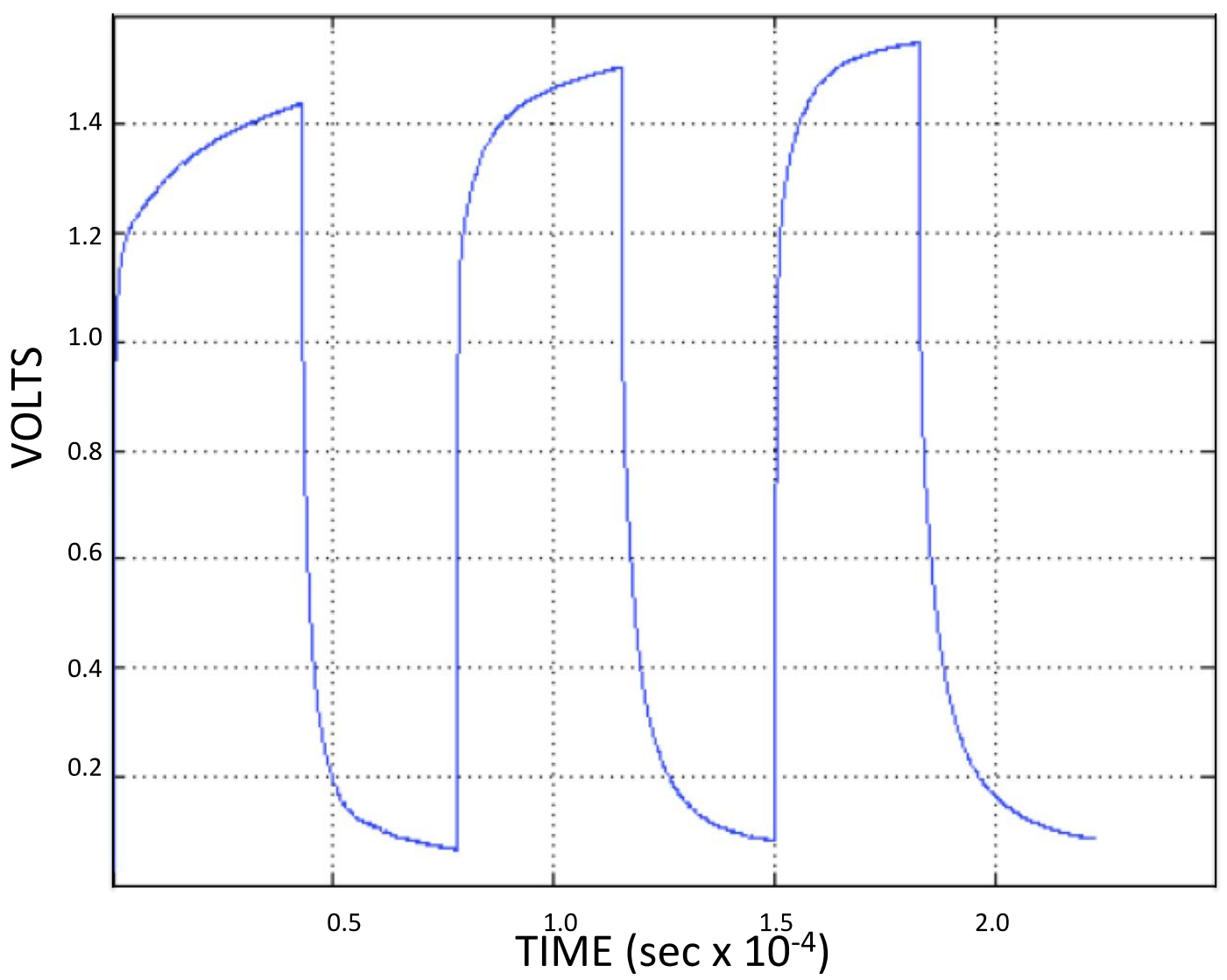

It is important to recognize the limitations of "Generation Zero" NPS-C created using A-SDM. First, they can only be charged to an ultimate voltage less than 2 Volts, irrespective of the applied charging voltage. Second, they only have a constant, high (SDM range) dielectric value, below about $0.8 \mathrm{~V}$. (This is similar to the "capacitive voltage" of a supercapacitor employing water as the electrolyte.) There is some small capacitance between the ultimate charging value (ca. $1.5 \mathrm{~V}$ ) and the onset of super dielectric behavior $(0.8 \mathrm{~V})$ but it does not contribute significantly to the energy storage capacity. Third, these materials (Generation Zero) are moderately unstable, as clearly all major aspects of behavior including ultimate voltage and dielectric constant change moderately (ca. 10\%) with each cycle. The magnitude of the instability is evident from an analysis of both the charging and discharging behavior of each part of the cycle. Using the highest voltage observed in each cycle for $\mathrm{V}_{0}$, the charging and discharge behavior is plotted in Figure 3, as per Equation (1). It is clear that the capacitive behavior changes with each cycle. 
Figure 3. Determination of aging on capacitive behavior. From Figure 2 cycle data charge and discharge constants can be derived. (a) The charge cycle data from Figure 2 is replotted using Equation (1), and shows clear regions of constant capacitance during charging; (b) the same procedure was applied to the discharge data from Figure 2.

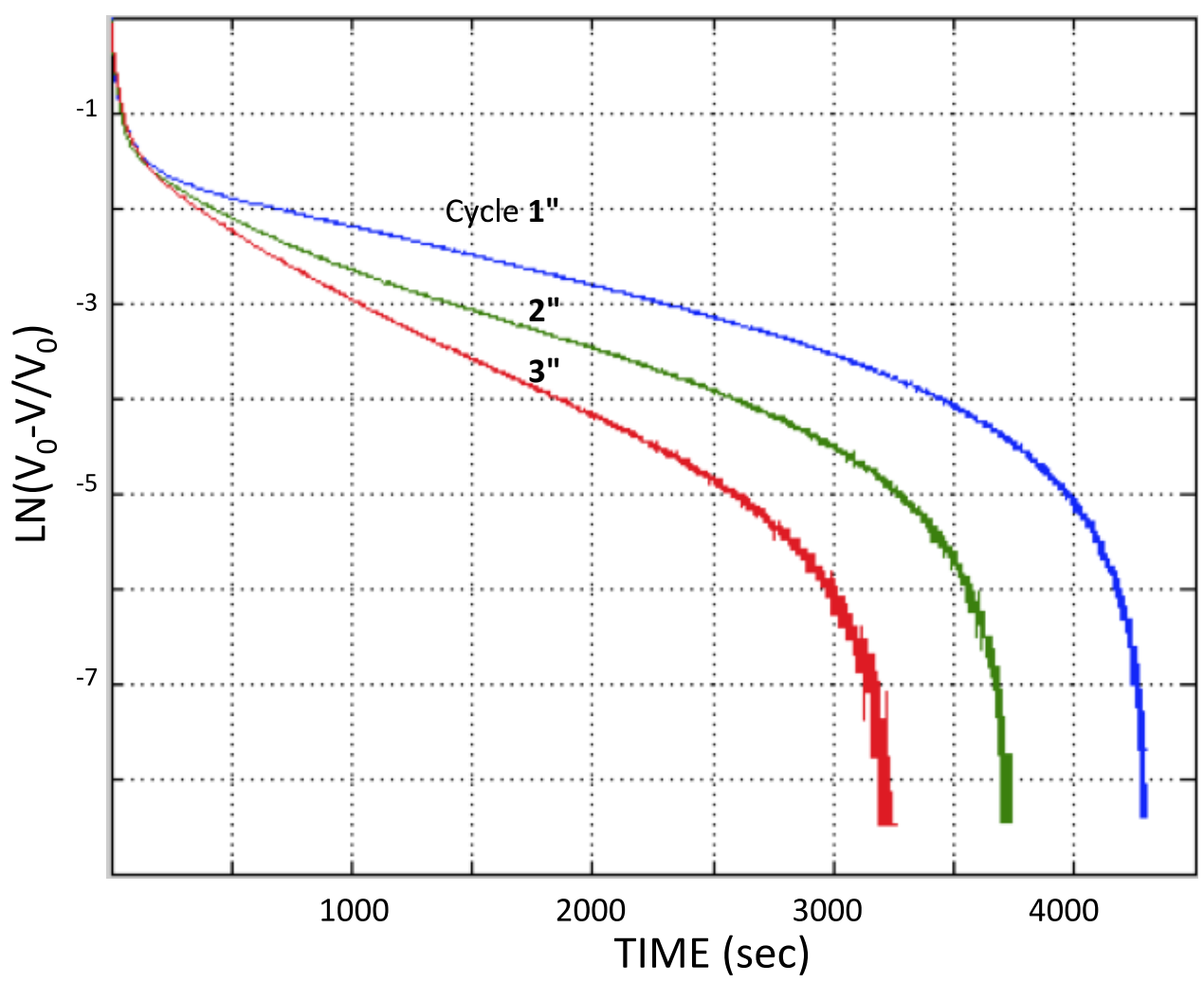

(a)

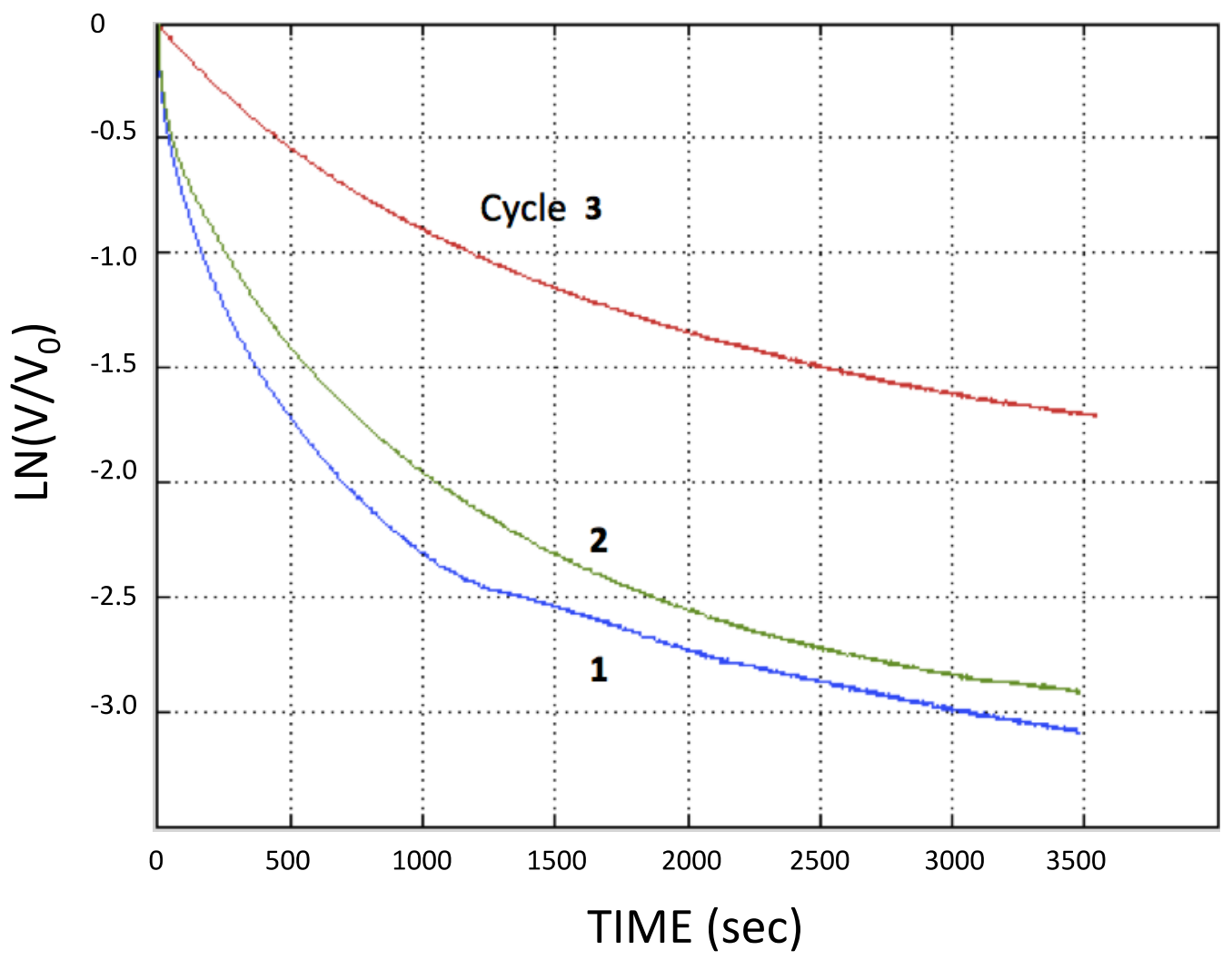

(b) 
The observed "instability", that is changing dielectric value, may result from gradual drying of the dielectric material. This postulate is consistent with one boundary condition repeatedly found: Approximately 10 days after creation, the capacitance of all samples went to zero. Physical examination of the dielectric material at this point showed it to be dry and cracked, in clear contrast to the initial wet, smooth, pasty consistency of the newly made capacitors. Moreover, it was repeatedly found that the careful addition of water to the dry, zero capacitance, dielectric material restored most of the original capacitive value. A simple hypothesis is consistent with the observations of (i) an initially increasing dielectric constant; and (ii) an eventual decrease to zero. To wit: There is an optimum amount, from the perspective of dielectric constant, of water in the A-SDM material.

Discharge behavior for the thicker, hence lower total capacitance, samples was found to show behavior more like that anticipated for classic capacitors. That is, discharge through a $528 \mathrm{kOhm}$ resistor, re-plotted per Equation (1), was more linear (Figure 4) than that observed through a $99 \mathrm{kOhm}$ resistor (Figure 3). This was not found to be the case for the thinner, higher capacitance samples. These samples displayed linear, constant dielectric behavior even for discharge through a $20 \mathrm{kOhm}$ resistor.

Figure 4. Dielectric constant through large load. The discharge data through a $528 \mathrm{kOhm}$ resistor for four alumina/boric acid super dielectric material (SDM) capacitors was fitted using Equation (2). It is clear that the linear part of a plot of $\ln \left(\mathrm{V} / \mathrm{V}_{0}\right)$ vs. time will yield the dielectric constant.

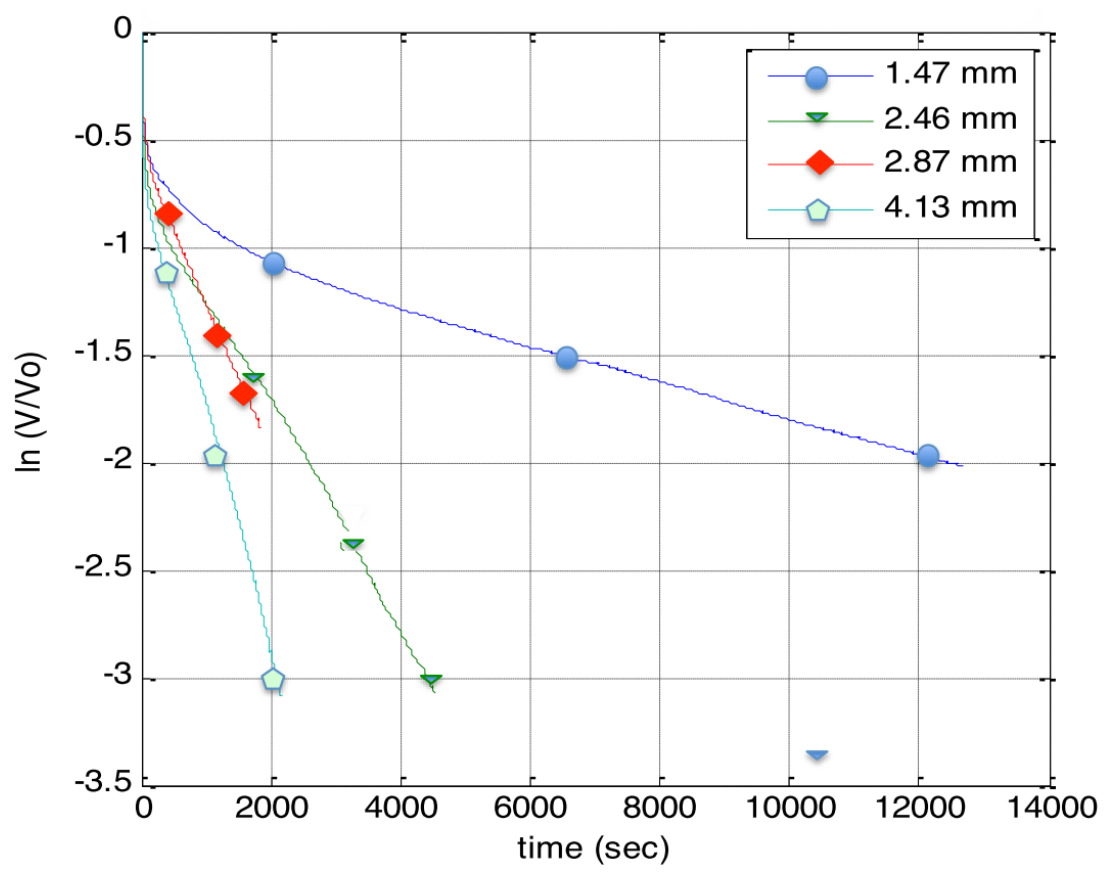

Only one parameter of the NPS-C was systematically modified in this (initial) study: the thickness of the A-SDM dielectric layer. As shown in Table 1, NPS-C capacitors with seven different thicknesses of the dielectric material were created and tested. The plotted data (Figure 5) shows that the capacitance, first discharge cycle only, increases with decreasing thickness. From a fit of the line, the capacitance can be projected to any thickness, and that value employed to predict energy density as a function of thickness. 
Table 1. Impact of thickness. Data in the first four rows shown correspond to Figure 3, hence discharge was through a $528 \mathrm{KOhm}$ resistor, and the last two rows through a $20 \mathrm{kOhm}$ resistor. As shown, the thinner the dielectric layer the higher the capacitance. It is also clear that the lower the "resistor" the higher the capacitance. Variation in measured dielectric constant possibly reflects the irregularity of handmade construction.

\begin{tabular}{cccccc}
\hline Test & $\begin{array}{c}\text { Dielectric } \\
\text { thickness }(\boldsymbol{d})\end{array}$ & $\begin{array}{c}\text { Initial } \\
\text { discharge } \\
\text { voltage }\left(\boldsymbol{V}_{\boldsymbol{0}}\right)\end{array}$ & $\begin{array}{c}\text { Dielectric constant } \\
\left(\boldsymbol{\varepsilon}_{\boldsymbol{R}}\right) \text { at operating } \\
\text { voltage }\end{array}$ & $\begin{array}{c}\text { Operating } \\
\text { voltage }\end{array}$ & $\begin{array}{c}\text { Dielectric } \\
\text { constant }(\boldsymbol{\varepsilon} \boldsymbol{R}) \text { over } \\
\text { entire range }\end{array}$ \\
\hline Discharge only $(528 \mathrm{k} \Omega)$ & $1.47 \mathrm{~mm}$ & $2.20 \mathrm{~V}$ & $1.81 \times 10^{9}$ & $0.7 \mathrm{~V}$ & $8.02 \times 10^{8}$ \\
Discharge only $(528 \mathrm{k} \Omega)$ & $2.46 \mathrm{~mm}$ & $2.16 \mathrm{~V}$ & $5.78 \times 10^{8}$ & $0.8 \mathrm{~V}$ & $3.52 \times 10^{8}$ \\
Discharge only $(528 \mathrm{k} \Omega)$ & $2.87 \mathrm{~mm}$ & $1.85 \mathrm{~V}$ & $4.44 \times 10^{8}$ & $0.9 \mathrm{~V}$ & $2.66 \times 10^{8}$ \\
Discharge only $(528 \mathrm{k} \Omega)$ & $4.13 \mathrm{~mm}$ & $2.18 \mathrm{~V}$ & $4.43 \times 10^{8}$ & $0.8 \mathrm{~V}$ & $2.86 \times 10^{8}$ \\
Discharge only $(99 \mathrm{k} \Omega)$ & $2.59 \mathrm{~mm}$ & $1.43 \mathrm{~V}$ & $5.0 \times 10^{9}$ & $0.2 \mathrm{~V}$ & $1.2 \times 10^{9}$ \\
Discharge only $(20.8 \mathrm{k} \Omega)$ & $0.38 \mathrm{~mm}$ & $1.60 \mathrm{~V}$ & $1.27 \times 10^{9}$ & $0.55 \mathrm{~V}$ & - \\
Discharge only $(20.8 \mathrm{k} \Omega)$ & $0.25 \mathrm{~mm}$ & $1.44 \mathrm{~V}$ & $1.54 \times 10^{9}$ & $0.6 \mathrm{~V}$ & - \\
\hline
\end{tabular}

Figure 5. Impact of thickness. Ideally (Equation (2)), there is an inverse relationship between capacitance and thickness, $d$. As shown, a linear fit of the data is consistent with theory. Note: the error bars for the "thick" dielectric capacitors are not shown as the errors are of the same size as the icons.

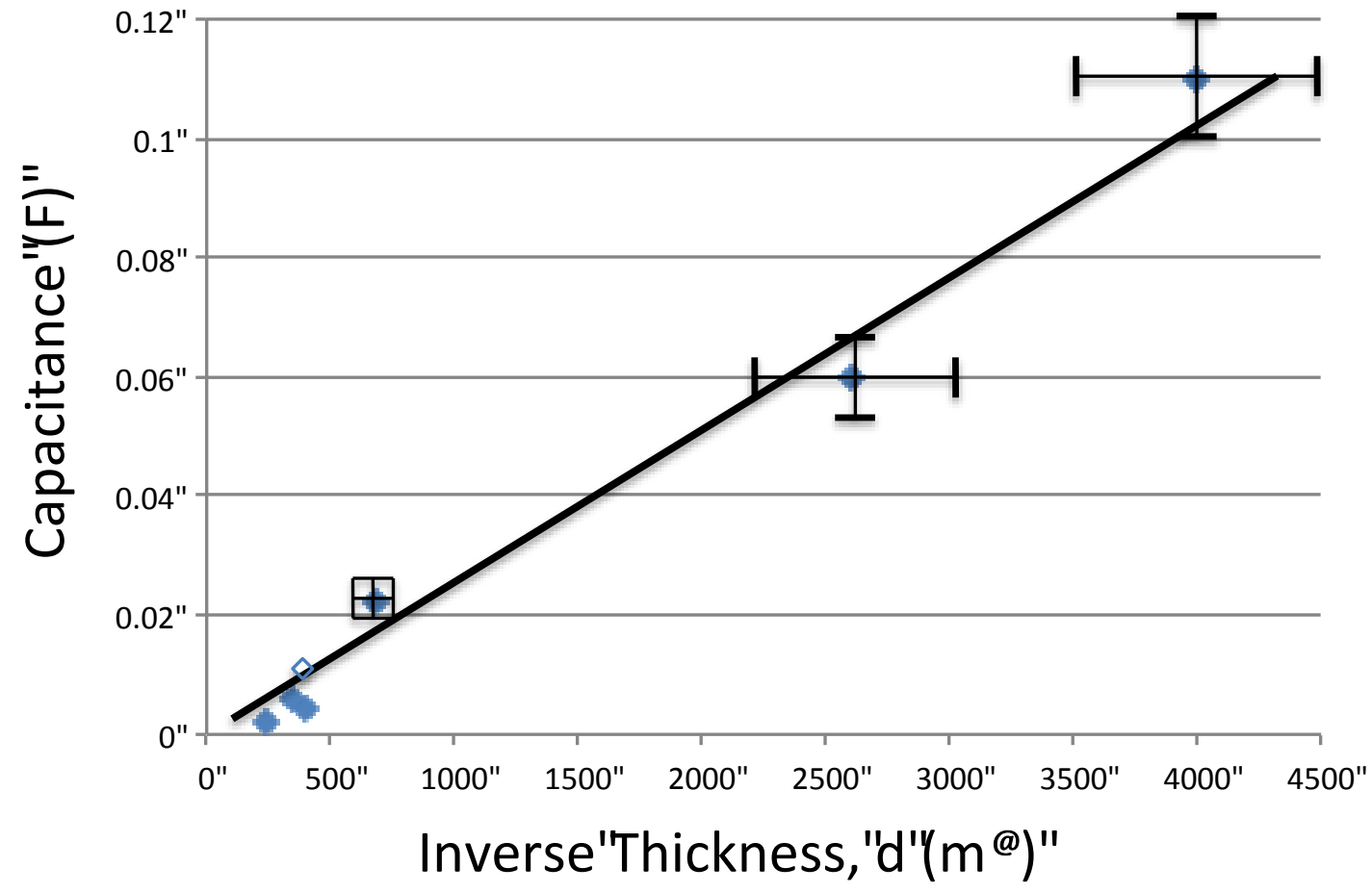

From the fitted line we obtain a nominal dielectric constant for the particular alumina chosen, for the particular water/boric acid/alumina ratio employed in this work (see Experimental Section), $\sim 1 \times 10^{9}$, clearly making this a "superdielectric" below about $0.7 \pm-0.2 \mathrm{~V}$. 


\section{Discussion}

The most important result of this work is the demonstration of the existence of a new class of dielectrics, super dielectric materials, with dielectric constants, near $0 \mathrm{~Hz}$, greater than $10^{5}$. The specific dielectric employed was a high surface area alumina impregnated, to the consistency of paste, with an aqueous solution of weak boric acid (A-SDM). This material repeatedly showed dielectric constants greater than $4 \times 10^{8}$. Thus, the data are consistent with the underlying application postulate: Adding a concentrated ionic solution to highly porous insulating materials creates a high dielectric material. Moreover, assuming a thickness of 5 micron, a typical value for inexpensive commercial ceramic capacitors, and using a rounded "average" dielectric constant of $1 \times 10^{9}$ (see Table 1, Figure 5), yields a remarkable energy density of approximately $1000 \mathrm{~J} / \mathrm{cm}^{3}$ at $2.5 \mathrm{~V}$. The voltage selected, $2.5 \mathrm{~V}$, is typical for the breakdown of the best electrolytes. Even a computation based on the lowest dielectric constant $\left(4.4 \times 10^{8}\right)$ and the lowest "capacitive voltage" $(0.7 \mathrm{~V})$ measured in the present work, leads to an energy density of $\sim 40 \mathrm{~J} / \mathrm{cm}^{3}$, still a remarkable value.

The phenomena observed are all consistent with the hypothesis/model stated in the Introduction: Charged species in liquid drops in the pores of solids will migrate to create dipoles, equal in size to the drops, in an applied electric field. The hypothesis was initially proposed on the basis of standard aspects of dielectric theory, in particular the understanding that the best solid dielectrics, such as barium titanate, are superior because of the magnitude and density of dipoles that form in an applied electric field. The greater the magnitude and density of dipoles, the better the dielectric. That is, the model employed is simply a variation on the most classic model of dielectric behavior, the so-called polarizable (or "ponderable") media model [22].

In the A-SDM it is postulated charge separation, leading to dipole formation, occurs in the nano-scale drops of ion containing liquid in the pores of the alumina. Specifically, in an applied electric field the positive ions will tend to cluster toward the capacitor cathode, and negative ions toward the anode. This creates a greater charge separation, physically longer, and probably larger, than is possible in a solid crystal. Indeed, charge motion in a solid crystal is physically limited to a very small distance, in fact less than the diameter of an atom. In a water drop, charge separation can occur over the entire length of a pore. Moreover, in solids the positive ions cannot move, whereas in a liquid, both negatively and positively charged ions can migrate, increasing the dipole moment.

In particular, the model is consistent with these observations. First, at a voltage of around one volt, there is a "dielectric breakdown" of water. The water does not only exist in the pores, but forms a matrix that fills all the empty space within the powder, once this breakdown occurs, there is a conduction path from cathode to anode. Hence, at voltages above the breakdown voltage the effective dielectric constant drops quickly to zero, as observed.

The value of breakdown can be estimated. These three values enable a calculation: (i) The breakdown voltage of distilled water is $\sim 65 \times 10^{6} \mathrm{~V} / \mathrm{m}$ [23]; (ii) ionic solutions break down at a voltage between $10 \%$ and $30 \%$ lower than distilled water [24]; (iii) the average pore size in the alumina is approximately $250 \AA$, or $25 \times 10^{-9} \mathrm{~m}$. Assuming that a boric acid solution sustains $30 \%$ less voltage than water these values indicate that the average drop should not be able to sustain a voltage greater than $\sim 1.1 \mathrm{~V}$. This is reasonably close to the observed value of $\sim 0.8 \mathrm{~V}$. 
Even in the absence of an overall discharge path between electrodes, each individual drop would no longer be able to support a charge separation above the breakdown voltage. Second, in the absence of water there are no liquid drops available to form dipoles. This is consistent with the virtual disappearance of any capacitance once the dielectric fully dries. Third, once water is added to a desiccated dielectric the drops can reform, the chargeable species will still be present (probably on the pore walls), and will re-dissolve, hence, the original dielectric behavior will be observed.

A rough case can also be made that the large dielectric constants observed are quantitatively consistent with the model. In the case of the alumina employed herein, BET analysis indicated the average pore radius was of the order $250 \AA$. This indicates that the average dipoles are of length of the order $500 \AA$. As the electric dipole moment is proportional to the charge separation distance, and the potential energy is proportional to the magnitude of the dipole, the energy is proportional to the charge separation. Moreover, the dipole moment is proportional to the amount of charge separated. A rough calculation is possible in the present case. Postulate: the "polarizability" of a media is proportional to the size of the dipoles (charge x separation length) times the density of dipoles. This is expressed as:

Dielectric constant $\alpha$ Dipole Length $\times$ Dipole Charge $\times$ Dipole Density

Given a pore diameter of $500 \AA$ (see Experimental section), and a charge separation in barium titanate of $0.1 \AA$, the SDM dipoles are about $5000 \times$ longer than those in barium titanate.

Second, it is easy to show that the total charge in a volume of barium titanate, and in the pores of a "water drop" of the same volume in an SDM are about the same. In a water drop we compute about $5 \times 10^{5}$ ions. The same volume of barium titanate contains about $10^{6}$ barium titanate primitive cells/dipoles. Finally, we assume the void space in imperfectly packed barium titanate matches the volume taken up by alumina.

Equation (2) for the SDM:

Dipole Constant/drop volume $\alpha 500 \AA \times 5 \times 10^{5}$ ions $\times 1$

For barium titanate:

Dipole Constant/equivalent drop volume $\alpha 0.1 \AA \times 1$ ion $\times 10^{6}$

The ratio of these two values suggests that SDM should have a dielectric constant more than 1000 times greater than barium titanate.

The simple model described above discussed above is only intended to be "approximate". It is clearly not fully quantitative as it suggests a three order of magnitude increase in dielectric constant relative to barium titanate, where the measured increase is at least two orders of magnitude greater.

Some speculative comments are useful for future model development. In particular, it is possible that on this scale each drop acts as a nearly perfect conductor, such that charges move until the field inside the drop is cancelled. This would make the drops "metal like", with nearly infinite permittivity. The combination of very large physical dipoles, of nearly infinite permittivity, might create the super dielectric constant values observed in the present work.

Some future experimental work is suggested for testing aspects of the model. For example, measurement of capacitance as a function of temperature could be useful. If the model is qualitatively correct, the capacitance should decrease with increasing temperature, and increase with decreasing 
temperature, until the drops solidify (freeze), at which point the dielectric constant should drop dramatically.

Other aspects of the model can be tested as well. For example the model suggests larger pores may produce higher dielectric values. Certainly, there are many alumina materials with different pore sizes readily available for testing this objectively. It is possible that the addition of small amounts of metal (below percolation threshold), by some as yet to be determined method, to a material with large pores will create a super dielectric material as metal has a nearly infinite permittivity.

Many objections to the proposed model will inevitably be raised. One likely objection is that water cannot enter pores below a certain size due to surface tension. Technically, this is correct, however; it is well known that water in the form of water vapor will enter pores of any size. In the event one or more "primary adsorption sites" (PAH) exist in the pore, the water molecule will adsorb and nucleate the formation of a drop of water from other vapor phase water molecules. This leads to Type III isotherm behavior and the complete filling of the pore at a vapor pressure equal to about $50 \%$ relative humidity [25-27]. As the wetted alumina should have a local RH of nearly $100 \%$, and there is a high density of PAH on a hydrophilic alumina surface, all pores, any size, should be filled. Future studies may test the SDM hypothesis through the use of alumina treated to create a hydrophobic surface. Such material should not exhibit SDM behavior.

It is also clear that that the data and model presented herein will not lead to an end to discussions of the origins of "large" dielectric constants. Earlier models were used to describe other demonstrations of high values of dielectric constant. For example, standard dielectrics, particularly barium titianate to which particles of metal have been added [8,28-30] have dielectric constants nearly ten times larger than the host ceramic [29]. Some models suggested the behavior arose from special characteristics of nano-metal particles [31-33], but this appears to be inconsistent with later experimental work that employed 50 micron Ni particles to achieve a similar outcome [29]. In any event, these models do not appear relevant to the current case as no metal of any sort is present in the dielectric.

Another class of models for unusually high dielectric constants, particularly for those observed for mixtures containing metal particles, are "percolation" models [34-36]. Purportedly, some material properties, in particular the dielectric constant, will diverge as the loading of the minority material (metal particles in this case) approaches the percolation limit of approximately $27 \%$ by volume [37]. These models do not appear to bear on the current observations as it is reasonable to presume that a robust percolation path always exists: (i) porous alumina, often used as a catalyst support, does not consist of closed pores; (ii) even in the "incipient wetness" mode, the material is sufficiently wet that a path between electrodes, external to the alumina particles, always exists. It is also notable that the percolation models require a precise "hit" of the percolation limit. This seems unlikely in the system described. Indeed, superdielectric behavior is observed over days, even as the amount of water in the system is clearly changing.

A final comment is reserved for a possible connection between the present work and the previously observed phenomenon of "colossal dielectric values" [10-14]. The origin of colossal dielectric constants is a matter of considerable discussion, and cannot be fully addressed here. One consensus is that the intrinsic dielectric properties of these materials are not "colossal" and that the colossal values arise from a range of extrinsic properties such as the high dielectric values of interfaces, including interface between dielectric and electrode and grain boundaries [14,38]. Other extrinsic high dielectric 
sources derive from the ability of trapped molecules, including water, to diffuse in porous networks in response to changing fields. Apparently, the process of production of some of these materials can increase the extrinsic dielectric value significantly [13].

There is one model of the origin of colossal dielectric constants, one not frequently cited, that is similar to the model postulated herein, and to the classic polarizable media model. To wit: "giant dipoles" form in some part of the solid dielectric media [38,39], resulting in colossal dielectric behavior.

Finally, it is interesting to speculate on the potential value of NPS capacitors. As noted earlier, reasonable extrapolation of the collected data, and the projected use of a dielectric with a higher discharge voltage, leads to a remarkable energy density of $\sim 1000 \mathrm{~J} / \mathrm{cm}^{3}$. A D-battery ("flashlight battery") has a volume of $\sim 53 \mathrm{~cm}^{3}$. Assuming that about half that volume is taken up by SDM of 5 micron thickness, the rest of the volume evenly divided between electrode and insulating layers, means a 1 D-cell sized NPS capacitor could hold 25,000 J. In contrast, the best "d-cell" supercapacitors (costly) advertise a capacitance of $3000 \mathrm{~F}$ and a voltage of $2.7 \mathrm{~V}$, for a total energy of approximately $11,000 \mathrm{~J}$. Could an NPS capacitor compete against a battery? A typical D battery can deliver just over $80,000 \mathrm{~J}$. This in turn suggests that with optimization, NPS capacitors, made of remarkably inexpensive material, could surpass supercapacitors, and rival batteries in terms of volumetric energy density.

\section{Conclusions}

The primary finding in this paper is empirical: A high surface area, highly porous, alumina filled to the point of incipient wetness with a weak aqueous boric acid solution has a dielectric constant $>10^{8}$. This value is at least three orders of magnitude greater than any previously recorded, and suggests this "multi-material" dielectric can be used to create electrostatic capacitors with energy density far better than the best EDLC.

There are a couple of significant caveats that suggest some areas of focus of future study. First, the present range of validity of this finding is restricted to very low frequency, of the order $10^{-2} \mathrm{~Hz}$. This data is suitable for assessing potential energy storage applications, but not the use of capacitors based on this material in circuit applications. It is notable, however; that preliminary data reported to the authors [40], based on the use of impedance spectroscopy, indicated that the unprecedented dielectric values persist to at least $1 \mathrm{kHz}$. Second, the material has an effective maximum operating voltage of $\sim 1 \mathrm{~V}$. Similar voltage restrictions are found in EDLC and for the same reason, electric breakdown in the electrolyte phase.

Finally, the data is completely consistent with a broad theory of "super dielectric" materials: Adding a concentrated ionic solution to highly porous insulating materials creates a high dielectric material. Further testing of this postulate is underway using $\mathrm{pH} 7$ salt solutions.

\section{Acknowledgments}

Partial support for this work was obtained from the Energy Expeditionary Office of the US Marine Corporation. 


\section{Author Contributions}

The experimental work for this paper was primarily the responsibility of CMDR Sam Fromille, US Navy and formed the core of his MS thesis. The work was organized and directed by Jonathan Phillips.

\section{Conflicts of Interest}

The authors declare no conflict of interest.

\section{References}

1. Jayalakshmi, M.; Balasubramanian, K. Review: Simple capacitors to supercapacitors. Int. J. Electrochem. Sci. 2008, 3, 1196-1217.

2. Stroller, M.D.; Park, S.; Zhu, Y.; An, J.; Ruoff, R.S. Graphene based ultracapacitors. Nano Lett. 2008, 8, 3498-3502.

3. Liu, C.; Neff, D.; Zhamu, A.; Zhang, B.Z. Graphene based supercapacitors with an ultrahigh energy density. Nano Lett. 2010, 10, 4863-4868.

4. Wang, Y.; Shi, Z.; Ma, Y.; Wang, C.; Chen, C.Y. Supercapacitor devices based on graphene materials. J. Phys. Chem. C 2009, 113, 13103-13107.

5. Kinoshita, K.; Yamaji, A. Grain-size effects on dielectric properties in barium titanate ceramics. J. Appl. Phys. 1976, 47, 371-373.

6. Arlt, G.; Hennings, D. Dielectric properties of fine-grained barium titanate ceramics. J. Appl. Phys. 1985, 58, 1619-1625.

7. Burfoot, J.C.; Taylor, G.W. Non-switching applications. In Polar Dielectrics and Their Applications; University of California Press: Los Angeles, CA, USA, 1979; p. 17.

8. El Kamel, F.; Gonon, P. Enhancement of the double-layer capacitance in $\mathrm{BaTiO}_{3}: \mathrm{H}-$ based solid electrolyte. J. Electrochem. Soc. 2010, 157, G91-G94.

9. Maglione, M.; Elissalde, C.; Chung, U. Interface control in $\mathrm{BaTiO}_{3}$ based supercapacitors. In Proceedings of the SPIE 7603 Oxide-based Materials and Devices, San Francisco, CA, USA, 23 January 2010; SPIE: Bellingham, WA, USA, 2010; p. 76030U.

10. Samara, A.A.; Hammetter, W.F.; Venturini, E.L. Temperature and frequency dependences of the dielectric properties of $\mathrm{YBa}_{2} \mathrm{Cu}_{3} \mathrm{O}_{6+x}$. Phys. Rev. B 1990, 41, 8974, doi:10.1103/PhysRevB.41.8974.

11. Rey, C.M.; Mathias, H.; Testardi, L.R.; Skirius, S. High dielectric constant and nonlinear electric response in nonmetallic $\mathrm{YBa}_{2} \mathrm{Cu}_{3} \mathrm{O}_{6+x}$. Phys. Rev. B 1992, 45, 10639-10646.

12. Lunkenheimer, P.; Fichtl, R.; Ebbinghaus, S.G.; Loidl, A. Nonintrinsic origin of the colossal dielectric constant in $\mathrm{CaCu}_{3} \mathrm{Ti}_{4} \mathrm{O}_{12}$. Phys. Rev. B 2004, 70, 172102:1-172102:4.

13. Yang, Y.; Wang, X.; Liu, B. $\mathrm{CaCu}_{3} \mathrm{Ti}_{4} \mathrm{O}_{12}$ ceramics from different methods. J. Mat. Sci. Mater. Electron. 2014, 25, 146-151.

14. Lunkenheimer, P.; Krohns, S.; Riegg, S.; Ebbinghaus, S.G.; Reller, A.; Loidl, A. Colossal dielectric constants in transition-metal oxides. Eur. Phys. J. 2009, 180, 61-89.

15. Anderson, J.C. Dielectric Breakdown. In Dielectrics; Reinhold Publishing Corporation: New York, NY, USA, 1964; p. 8. 
16. Ho, J.; Jow, T.R.; Boggs, S. Historical introduction to capacitor technology. IEEE Electr. Insul. Mag. 2010, 26, 20-25.

17. Phillips, J.; Clausen, B.; Dumesic, J.A. Iron pentacarbonyl decomposition over grafoil I: Production of small metallic iron particles. J. Phys. Chem. 1980, 84, 1814-1822.

18. Phillips, J.; Dumesic, J.A. Iron pentacarbonyl decomposition over grafoil II: Effect of sample outgassing on decomposition kinetics. Appl. Surf. Sci. 1981, 7, 215-230.

19. Reynolds, G.J.; Krutzer, M.; Dubs, M.; Felzer, H.; Mamazza, R. Electrical properties of thin film capacitors fabricated using high temeprature sputtered modified barium titanate. Materials 2012, $5,644-660$.

20. Barsoukov, E.; MacDonald, J. Impedance Spectroscopy Theory, Experimental and Applications, 2nd ed; John Wiley and Sons: New York, NY, USA, 2005.

21. Raistrick, I.D. Theory. In Impedance Spectroscopy. Emphasizing Solid Materials and Systems; Macdonald, J.R., Ed.; John Wiley and Sons: New York, NY, USA, 1987.

22. Jackson, J.D. Classical Electrodynamics; John Wiley and Sons: New York, NY, USA, 1975.

23. CRC Handbook of Chemistry and Physics, 91st ed.; CRC Press: London, UK, 2013.

24. Toriyama, Y.; Shinohara, U. Electric breakdown of water and aqueous solutions. Phys. Rev. 1937, 51, 680, doi:10.1103/PhysRev.51.680.

25. Phillips, J.; Kelly, J.D.; Radovic, L.; Xie, F. Microcalorimetry study of the influence of surface chemistry on the adsorption of water by high surface area carbons. J. Phys. Chem. B 2000, 104, 8170-8176.

26. Müller, E.A.; Rull, L.F.; Vega, L.F.; Gubbins, K.E. Adsorption of water on activated carbons: A molecular simulation study. J. Phys. Chem. 1996, 100, 1189-1196.

27. Dubinin, M.M.; Serpinsky, V.V. Isotherm equation for water vapor adsorption by microporous carbonaceous adsorbents. Carbon 1981, 19, 402-403.

28. Cheng, B.; Lin, Y.-H.; Yang, H.; Lan, J.; Nan, C.-W.; Xiao, X.; He, J. High dielectric permittivity behavior in Cu-doped $\mathrm{CaTiO}_{3}$. J. Am. Chem. Soc. 2009, 92, 2776-2779.

29. Pecharroman, C.; Esteban-Begegon, F.; Bartolome, J.F.; Lopez-Esteban, S.; Moya, J. New percolative $\mathrm{BaTiO}_{3}-\mathrm{Ni}$ composites with a high and frequency independent dielectric constant. Adv. Mat. 2001, 13, 1541-1544.

30. Pecharroman, C.; Esteban-Betegon, F.; Jimenez, R. Electric field enhancement and conduction mechanisms in $\mathrm{Ni} / \mathrm{BaTiO}_{3}$ percolative composites. Ferroelectrics 2010, 400, 81-88.

31. Saha, S.K. Observation of giant dielectric constant in an assembly of ultrafine Ag particles. Phys. Rev. B 2004, 69, 125416, doi:10.1103/PhysRevB.69.125416.

32. Valant, M.; Dakskobler, A.; Ambrozic, M.; Kosmac, T. Giant permittivity phenomena in layered $\mathrm{BaTiO}_{3}-\mathrm{Ni}$ composites. J. Eur. Ceram. Soc. 2006, 26, 891-896.

33. Gor'kov, L.P.; Éliashberg, G.M. Minute metallic particles in an electromagnetic field. Sov. Phys. JETP 1965, 21, 940-947.

34. Bergman, D.J.; Imry, Y. Critical Behavior of the complex dielectric constant near the percolation thereshold of a heterogeneous material. Phys. Rev. Lett. 1977, 39, 1222-1225.

35. Efros, A.L.; Shkolvskii, B.I. Critical behavior of conductivity and dielectric constant near the metal-non-metal transition threshold. Phys. Stat. Sol. B 1976, 76, 475-485. 
36. Efros, A.L. High volumetric capacitance near the insulator-metal percolation transition. Phys. Rev. B 2011, 84, 155134:1-155134:10.

37. Gerenrot, D.; Berlyand, L.; Phillips, J. Random network model for heat transfer in high contrast composite materials. IEEE Trans. Adv. Packag. 2003, 26, 410-416.

38. Lunkenheimer, P.; Bobnar, V.; Pronin, A.V.; Ritus, A.I.; Volkov, A.A.; Lodl, A. Origin of apparent colossal dielectric constants. Phys. Rev. B 2002, 66, 052105:1-052105:4.

39. Müller, K.A. Phase Separation in Cuprate Superconductors; Sigmund, E., Müller, K.A., Eds.; Springer: Berlin, Germany, 1994; p. 1.

40. Yun, L. (Australia National University, Canberra, Australia). Values Based on Impedance Spectroscopic Investigation of Capacitors Generated According to the Authors Specification and Using Author Supplied Material. Personal Communication, 2014.

(C) 2014 by the authors; licensee MDPI, Basel, Switzerland. This article is an open access article distributed under the terms and conditions of the Creative Commons Attribution license (http://creativecommons.org/licenses/by/4.0/). 